\title{
Information training in physics course
}

Ding-yu Liu, Hong-zhi Bao

Ding-yu Liu, Hong-zhi Bao, "Information training in physics course," Proc. SPIE 9663, Eighth International Topical Meeting on Education and Training in Optics and Photonics, 96630W (6 October 2003); doi: 10.1117/12.2208453

SPIE Event: Eighth International Topical Meeting on Education and Training in Optics and Photonics, 2003, Tucson, Arizona, United States 


\title{
Information training in physics course
}

\author{
Liu Ding-yu and Bao Hong-zhi \\ Department of Mathematics and Physics, Dalian Maritime University \\ physical laboratory, Dalian maritime university, Liaoning province, PRC 116026 Dalian China \\ telephone: 086-0411-4729308; e-mail: baohongzhi1@sina.com
}

\begin{abstract}
Three levels of NPMT: (A) NPMT training for the first-and-second-year students; (B) NPMT training for higher level student; (C) NPMT training for postgraduate students. (C)2003 Optical Society of America

OCIS codes: (070.4340) Nonlinear optical signal processing; (230.4320) Nonlinear optical devices
\end{abstract}

\section{Introduction}

The information is as one of the three elements of the system to be constructed as substance and energy, since the development of Information Theory in the last century. Up to date, however, the conception of information has not been introduced into university physics and its experimental training, The reason may be originated from the statistical definition of entropy that can not be taught in advance. But according to the general definition that "Information is an object for transmitting and processing which is loading on some data, signals and messages." Such definition can be accepted and understood by the first-and-second-year students in any colleges and universities. Since the conception of information is widely used for many modern scientific field and technologies. For examples:

1.It can be used for early diagnosis of lung cancer, ${ }^{1,2}$ which is of great importance to medical application. and other X-ray, CT and MRI diagnosis.

2.It can be used for keep a lookout over the degeneration of grazing land, for keep watch on pollution of environment; for the report of fire alarm of forest, for topographic survey and the general configuration of the earth surface; for the remote sensing; etc.

As for physics training whatever mechanics, acoustics, electronics, optics and statistical thermodynamics, all of them can also be used for information transmitting and processing,. However, we should take electro-optical technique into consideration first and foremost, because of its widespread influence in modern sciences and technologies. We have found that optical experiments for training are not beyond the scope of traditional features, after some investigation of universities around our nation, such as the measurement of wavelength by diffraction of grating; the measurement of thin film thickness by interference of two beams, take Michelson's experiment and Newton's ring for instance, and the measurement of Brewster angle etc. Besides. Holographic experiments for wave construction and reconstruction is the only one that has become a new technique training in some universities in China for about twenty years. But it needs vibration isolation workstation, and not in great demand.

According to the innovation demands of the Ministry of Higher Education and the development of electro-optics, based on our research work in the field of white-light information processing during 1986-1994,we select nonlinear phase modulation method of density pseudocolor encoding technique (NPMT for short) as our object for the innovation of experimental training in university physics because of the following advantages:

1. Satisfying the needs of the times of information.

2. It contains some fundamental ideas of information processing, such as linear phase and nonlinear phase modulation, encoding and decoding processing, spectrum constitution, , optical filtering operation, color mixing, the function of 4F-system. All of them are of universal significance.

3. Student will have some initial perceptual knowledge and know that how to put this knowledge to use.Since such conceptions are acquired by practical operation, careful measurement, and actual observation, so even the lower class student can accept them easily for qualitative understand .

4. On the other hand, It is not so easy to understand them by quantitative analysis. Therefore, we divided our subject into three levels for different class students. i..e.

A: Initial training for NPMT which can be taught for first-and -second-year students.

$\mathrm{B}$;Intermediate training which can be taught for higher class students.

C: Higher training for postgraduate students.

There are different teaching demands for the students of different levels.It may be shown as follows: 


\section{Three levels of NPMT}

\section{A: NPMT training for the first-and-second-year students.}

\section{A-1.Training purpose:}

a: Put black-and-white transparency to a pseudo color image with nonlinear phase modulation encoding and decoding techniques to provide better visual discriminition.

b: Learning the principle and functions of a nonlinear phase modulation pseudocolorimeter.

c: NPMT training results only for handling pseudo color encoding and decoding techniques qualitatively, not for quantitative analysis. .

A-2 Equipment:: a: nonlinear phase modulation pseudocolorimeter, (made in Dalian maritime university) b: dark room with processing kit (ready for use) c: Ronchi grating, d: cassette (for encoding processing without dark condition), e: transparency with gray levels distribution ( to be pseudo color processing) f: H-D curve (for reference).

\section{A-3 Principle (only for lower class students) ${ }^{6}$}

Using the characteristic curve of the holographic emulsion as shown in FIG.1. There are three portions, i.e. straight-line region, nonlinear region including gross fog region and saturation region. After developing and bleaching process, different photographic density will produce different relief thicknesses $\mathrm{d}$ where $\mathrm{d}$ is proportional to the photographic density $\mathrm{D}$, and can be represented in a simple relation

$$
\text { . . . } \quad \mathrm{d}=\beta \mathrm{D}
$$

the relief thickness will change the phase quantity of the incident light wave, which is referred to as the phase modulation. Two methods to be used, they are toe modulation and shoulder modulation. As for toe modulation, a fresh film or plate is used for light exposure with the $\log \mathrm{E}$ at toe point, after that, reserve the latent image without developing ready for the next exposure. .As for the shoulder modulation which is exposure closed contact with a Ronchi grating by appropriate exposure time. Both are called latent image firms or plates.

When a transparency is ready for pseudo color processing, take the latent image film or plate, put it to superimpose on the transpa-

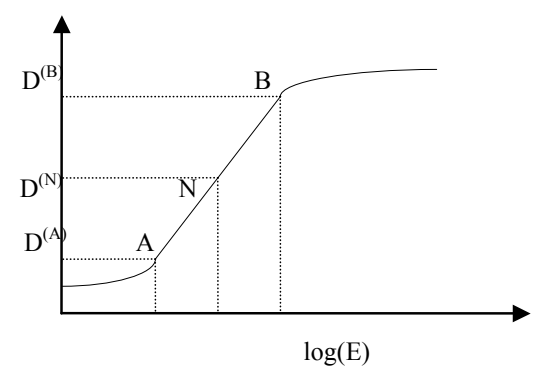

IG.1 Toe and shoulder point on H-D curve rency.. If the toe modulation film or plate is used, a Ronchi grating should be used to press on the transparency in order to protect the latent image part without secondary exposure, and let the light pass through the transparent part of the grating in order to receive secondary exposure. If the shoulder modulation film or plate is used, grating is not necessary. The exposures in both cases are falling onto the linear region of the H-D curve.. After developing and bleaching processing, the thickness difference produced by the latent image and that of the secondary exposure region is called effective thickness which may be denoted by the expression as

$$
, \quad . \quad \Delta \mathrm{d}_{\mathrm{j}}=\mathrm{d}-\mathrm{d}_{\mathrm{j}}=\beta \Delta \mathrm{D}_{\mathrm{j}}
$$

$\mathrm{j}=\mathrm{a}$ (for toe modulation), $\mathrm{j}=\mathrm{b}$ (for shoulder modulation). Since they are all used the nonlinear region of the H-D curve, so it is called nonlinear phase modulation. The relief corresponding to the latent image is produced only by the first exposure, so it is independent of the secondary exposure, and remains constant. During the period of secondary exposure, passing through the different gray parts of the transparency, the exposures are different due to different part of the linear region of the H_D curve. So they have different effective thickness. It should be noted that different gray levels on the transparency transferred into different 
effective thickness which produce different phase variations of the incident light wave.. .

Next, how to let the transparency of gray distribution transfer into color picture? The action of the grating ihas now been taken place. Using the principle of the diffraction of the white-lght. The relief grating is made by the simultaneously expose to the white light as shown in FIG.2 (for toe modulation)and FIG.3 (for shoulder modulation) Since the relief thickness whatever toe modulation or shoulder modulation remains constant, only the correspond to the different part of linear region of H_D curve can have different heights of the effective thickness to form a different phase grating. with different heights.. Using different height of phase grating to produce different colors by diffraction principle. Hence a color spectrum will be formed on the spatial frequency plane.

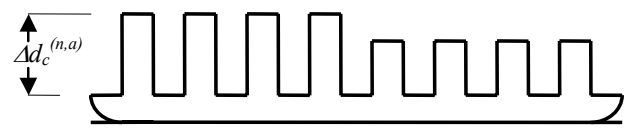

FIG.2 Rectangular model for toe modulation

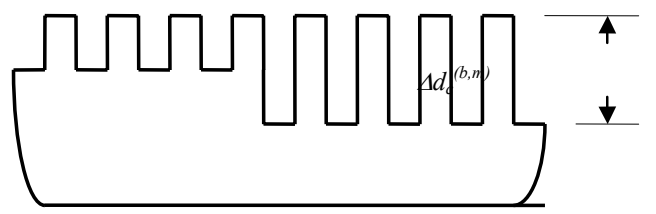

FIG.3Rectangular model for shoulder modulation

To resolve the white light of incidence by an encoding transparency with phase modulation, a typical equipment called 4F-system may be used as shown in FIG.4. In which the encoding transparency is inserted in the input plane $\mathrm{P}_{1}$. $\mathrm{P}_{1}$ is illuminated by a bundle of parallel white light through a collimating lens $\mathrm{L}_{1}$. The transmitting light fall on the focal plane of spatial frequency spectrum. through a Fourier lens $\mathrm{L}_{2}$.constituates a color spectrum. Such spectrum carries information of gray levels "dyed in colors." Different gray levels dyes in different colors.

.The function of the lens $\mathrm{L}_{2}$ is producing a diffraction pattern ( or say Fourier transformation mathematically, which may be denoted by the symbol F). If the encoding relief may be described by the functionf ( $\mathrm{x}, \mathrm{y})$ then the colorful spatial frequency spectrum may be described in general as the form

$$
p(\alpha, \beta)=\mathbf{F}[\mathrm{f}(\mathrm{x}, \mathrm{y})]
$$

where $\mathrm{p}(\alpha, \beta)$ is the function representing the colorful spatial frequency spectrum. So we have spectra of zeroth, first, second, third, etc., orders. The longer the wavelength, the larger the deviation for a given order of spectrum.. In order to control the color of the picture, we may select any order of spectra to go through the plane by open a small hole with appropriate size. Take zeroth order, for instance, open a small hole at the central position. Then the zeroth order spectrum will pass through the hole. This operation is referred to as optical filtering.

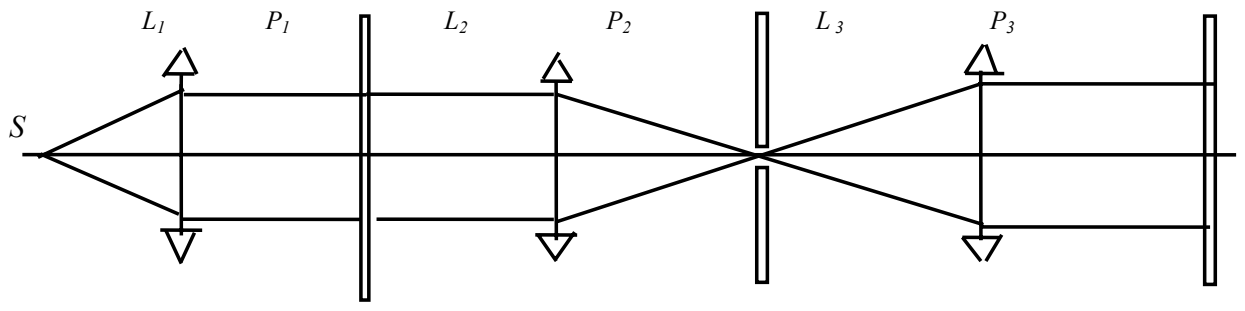

FIG.4 $4 F$-system of psudocolorimeter

In general, the filtering operation may be described by a filtering function $\mathrm{H}(\alpha, \beta)$ to represent the shape of the filtering aperture. then the light wave passes the aperture may be denoted by the function $\mathrm{p}(\alpha, \beta) \mathrm{H}(\alpha, \beta)$. which is the information "dyed in some colors" corresponding to the original gray levels distribution. It can be seem that we can reconstruct the original image, so long as we restore the information we have just obtained to the original shape but dyed with colorful information. Thus, the transformation from a black-and-white picture to a pseudo color image has been achieved. Since the black-and-white picture should be changed to a picture with photographic density distribution, hence it is referred to as density pseudocolor encoding technique. 
In order to reconstruct the original picture with pseudo colors, we may place a imaging lens behind the filtering plane as a focal plane of the lens $\mathrm{L}_{3}$ then the colorful image corresponding to the original black-and-white transparency will appear on the output plane of the $4 \mathrm{~F}$-system. In other words, $\mathrm{L}_{2}$ realized spatial frequency spectrum transformation, and on the contrary, $\mathrm{L}_{3}$ realized the inverse transformation. inverse Fourier transformation denoted by $\mathrm{F}^{-1}$ Now let $(\xi, \zeta)$ be the colorful coordinate, then the amplitude can be express as

$$
\mathrm{g}(\xi, \zeta)=\mathbf{F}^{-1}[\mathrm{p}(\alpha, \beta) \mathrm{H}(\alpha, \beta)]
$$

The intensity observation is then represented by the formula shown as follows:

$$
\mathrm{I}(\xi, \zeta)=/ \mathrm{g}(\xi, \zeta) /^{2}
$$

If we use the relief shape as shown in FIG.2m or FIG.3, illuminating with monochromatic wave, after a theoretical calculation we obtain the intensity formula as follows

$$
\mathrm{I}_{\lambda}(\xi, \zeta, \lambda)=\mathrm{K}_{1}+\mathrm{K}_{2} \cos [\mathrm{k}(\mu-1) \Delta \mathrm{d}]
$$

where $\mathrm{K}_{1}$ and $\mathrm{K}_{2}$ are constants, $\mathrm{k}$ is wave vector $2 \pi / \lambda \mu$ is the refractive index of the emulsion, $\Delta \mathrm{d}$ is the effective thickness of the uniform relief at $(\xi, \zeta$. . It should be noted that the above formulae are only applied to the case of monochromatic light. As a matter of fact, all monochromatic light passing through the filtering aperture are mixed together at output plane, and they are related to the power spectrum $\mathrm{G}(\lambda)$ of the light source Therefore, the intensity of the mixing colors should be express by the sum of various monochromatic light waves, i.e.

$$
\mathrm{P}(\xi, \zeta)=\sum_{\lambda} \mathrm{G}(\lambda) \mathrm{I}_{\lambda}(\xi, \zeta, \lambda) \Delta \lambda
$$

It should be noted that all formulae as mentioned above are only for qualitative interpretation, no concrete functions have been given. So it is only for qualitative understand by the first-and-second-year students. The quantitative analysis is beyond the requirement of the lower class student.

\section{A-4 : Experimental method (neglected)}

\section{B: NPMT training for higher level student}

\section{B-1. Training purpose:}

a: Making positive and negative relief.

b: As a matter of fact, the manufacture of positive and negative relief is the key to grasp the essentials of the nonlinear phase modulation pseudo color encoding technique.

B_2. Equipment: a: The same equipment as shown in A-2. b: Step exposure device. c: Interference microscope (for the measurement of relief thickness). d: Power meter (for the measurement of the transmittance). e: Holographic films or plates. f: Computer (or microprocesser) with software of relief calculation. :

\section{B-3 Principle for making positive and negative film and relief ${ }^{3,4}$}

\section{a: Making positive film with gray levels}

Using fresh film (or plate) for step exposure by step timer. The step exposure is defined as $\mathrm{E}_{\mathrm{s}}^{(\mathrm{n})}=\mathrm{nE}_{0}$, where $\mathrm{E}_{0}$ is the minimum exposure, $\mathrm{n}$ is an integer. Then we have a series of standard positive film (or plate). The photographic density difference of any two positive gray levels is

$$
\Delta \mathrm{D}_{\mathrm{s}}{ }^{(\mathrm{n}, \mathrm{m})}=\log (\mathrm{n} / \mathrm{m})^{\gamma}
$$

where $\mathrm{n}$ and $\mathrm{m}$ are the step exposure called exposure index $\mathrm{n}$ and $\mathrm{m}$ respectively, and the slop of the linear region is referred to as film $\gamma$.

\section{b: Making negative film with gray levels}

Using positive film (or plate) with step gray levels as attenuator superimpose upon a fresh film (or plate). The exposure is defined as $E_{c}^{(n)}=T_{s}^{(n)} E_{0}$, where $T_{s}^{(n)}$ is the nth transmittance of the positive gray levels. Then we have a series of negative step gray levels. with film $\gamma$ '. The photographic density difference of any two levels will be 


$$
\Delta \mathrm{D}_{\mathrm{c}}^{(\mathrm{n}, \mathrm{m})}=\gamma \gamma^{\prime} \log (\mathrm{m} / \mathrm{n})
$$

A simple relation of transmittance between positive and negative gray levels can be obtained as

$$
\mathrm{T}_{\mathrm{c}}^{(\mathrm{m})} / \mathrm{T}_{\mathrm{c}}^{(\mathrm{n})}=\left(\mathrm{T}_{\mathrm{s}}^{(\mathrm{n})} / \mathrm{T}_{\mathrm{s}}^{(\mathrm{m})}\right)^{\gamma^{\prime}}
$$

\section{c: Negative film with toe modulation:}

Using negative film (or plate) processed by toe modulation, we may put $\mathrm{m}=\mathrm{a}$, where a represents exposure index at toe point, then we have photographic density difference between toe point and any point along linear region ot H-D curve shown as follows

$$
\Delta \mathrm{D}_{\mathrm{c}}{ }^{(\mathrm{n}, \mathrm{a})}=\mathrm{D}_{\mathrm{c}}{ }^{(\mathrm{n})}-\mathrm{D}_{\mathrm{c}}{ }^{(\mathrm{a})}=\gamma^{\prime} \log \left(\mathrm{E}_{\mathrm{c}}{ }^{(\mathrm{n})} / \mathrm{E}_{\mathrm{c}}{ }^{(\mathrm{a})}\right)
$$

Since $E_{c}{ }^{(n)} / E_{c}^{(a)}=(p / n)^{\chi}$, and $E_{c}^{(n)}=E_{c}^{(a)}+E_{c}^{(n-a)}$ hence we have

$$
\Delta \mathrm{D}=\gamma^{\prime}\left[1+(\mathrm{p} / \mathrm{m})^{\gamma}\right]
$$

where $\mathrm{p}$ is the exposure index at toe, and $\mathrm{m}=\mathrm{n}-\mathrm{p}$ is the exposure index difference between toe and any exposure index along linear region of H-D curve.

\section{d: Negative film with shoulder modulation}

Using negative film (or plate) processed by shoulder modulation., we may put $n=b$, where $b$ represents exposure index at shoulder .point, then we have photographic density between shoulder point and any point along linear region of H-D curve, say m, shown as follows: .

$$
\Delta \mathrm{D}_{\mathrm{c}}^{(\mathrm{b}, \mathrm{m})}=\mathrm{D}_{\mathrm{c}}^{(\mathrm{b})}-\mathrm{D}_{\mathrm{c}}^{(\mathrm{m})}=\gamma^{\prime} \log \left(\mathrm{E}_{\mathrm{c}}^{(\mathrm{b})} / \mathrm{E}_{\mathrm{c}}^{(\mathrm{m})}\right)
$$

Since $E_{c}^{(b)} / E_{c}^{(m)}=(m / b)^{\gamma}$, then we have a simple relation

$$
\Delta \mathrm{D}_{\mathrm{c}}^{(\mathrm{b}, \mathrm{m})}=\gamma^{\prime} \gamma \log (\mathrm{m} / \mathrm{b})
$$

\section{B-4 Experimental method (neglected).}

\section{C: NPMT training for postgraduate students.}

\section{C-1 Training purpose:}

a: Based on the results of A and B, make a series of standard pseudo color plate with the pseudo color controller.

b: Make a software design for computing chromaticity coordinate of a series of standard pseudo colors.

C-2 Equipment: a: The same as shown in B-2, b: Color controller. c: Computer (or special microprocesser)

\section{C-3 Principle for pseudocolor analysis: ${ }^{5,7}$}

The approximate theory called rectangular model in A-3 has been briefly introduced before. This theory may be used for qualitative interpretation how to make pseudo color picture from a black-and-white transparency. This model is not able to give exactly the chromaticity coordinates. Some references should be read by the student himself because of the requirement of independent work.. The method for computing chromaticity coordinates of pseudo colors may be referred to the papers shown behind. The duty of a tutor or a professor is that guides the student to catch knowledge by his own brain and do not take on what ought to be done by studentt. So we 'd like say no more about it. .

C-4: Experimental method (neglected).

\section{References:}

1. Liu Ding-yu, "Color analysis of nonlinear phase modulation method for density pseudocolor encoding technique in medical application," Proceedings of SPIE-The International Society for Optical Engineering V1443 Feb 25 - 261991 p191 - 197.

2. Liu Ding-yu, "Optical specifications for pseudocolor diagnosis,"- Proceedings of SPIE - The International Society for Optical Engineering V 1896 Feb 14-19 1993 p397 - 402.

3. Liu Ding-yu, "Optical theory of psudocolor device for radiographic diagnosis with phase modulation," $<$ Soviet Chinese Joint Seminar “Holography and Optical Information Processing - Proceedings > Sept 21 - 261991 p28 - 32. 
4. Liu Ding-yu, sun Giang, "Theory of relief technique and color display," Proceedings of SPIE - The International Society for Optical Engineering V 2321 Supplementary Papers Aug 199415 - 18 p91-94.

5. Liu Ding-yu, <Electro-Optical Information Processing $>$ Chapter 13, Chapter 14-to be published in 2003.

Proc. of SPIE Vol. 9663 96630W-6

Downloaded From: https://www.spiedigitallibrary.org/conference-proceedings-of-spie on 26 Apr 2023 Terms of Use: https://www.spiedigitallibrary.org/terms-of-use 